\title{
COMPARISON OF INTRA-ARTICULAR METHYLPREDNISOLONE AND KETOROLAC INJECTIONS IN IMPROVING RANGE OF MOTION FOR DIFFERENT SHOULDER JOINT DISORDERS
}

\author{
Sumeera Matee, Wasif Anwar, Sami Wahid, Saeed Bin Ayaz*, Rana Shahid, Nadeem Ahmed Sheikh** \\ Combined Military Hospital Mangla/National University of Medical Sciences (NUMS) Pakistan, *Combined Military Hospital Jhelum/National University of \\ Medical Sciences (NUMS) Pakistan, ${ }^{* *}$ Combined Military Hospital Quetta/National University of Medical Sciences (NUMS) Pakistan
}

\begin{abstract}
Objective: To compare the efficacy of intra-articular injection of methyl prednisolone with ketorolac for improvement in range of motion in various shoulder joint disorders.

Study Design: Quasi-experimental study.

Place and Duration of Study: Departments of Internal and Rehabilitation Medicine, Combined Military Hospital Mangla, from Nov 2018 to May 2019.

Methodology: Through non-probability consecutive sampling, patients with shoulder disorders were enrolled in the study and divided into two groups. Group A received intra articular corticosteroid injection and group B received intra-articular Ketorolac injection. Outcome was measured in terms of improvement in shoulder range of motion.

Results: A total of 60 patients were selected, $40(66.7 \%)$ male and $20(33.3 \%)$ female. Thirty (50\%) patients had adhesive capsulitis, $24(40 \%)$ had rotator cuff syndrome and $6(10 \%)$ had impingement syndrome. Twenty four patients received methyl prednisolone acetate injection while 36 received ketorolac injection. There was no significant difference in the mean gain in flexion, extension, abduction, internal or external rotation between both groups $(p=0.224, p=0.261, p=0.884, p=0.238$, and $p=0.584$ respectively).

Conclusion: There was no significant difference in efficacy of corticosteroid and ketorolac when injected intra-articularly in shoulder joint disorders.
\end{abstract}

Keywords: Intraarticular injections, Methylprednisolone, Ketorolac, Shoulder joint.

This is an Open Access article distributed under the terms of the Creative Commons Attribution License (https://creativecommons.org/licenses/by-nc/4.0/), which permits unrestricted use, distribution, and reproduction in any medium, provided the original work is properly cited.

\section{INTRODUCTION}

Shoulder joint has unique dynamics. This function is attributed to its ball and socket nature. Tasks such as prehensile function of hand, reach, and exploration of surroundings are basic for survival. The extensive ranges of motion of shoulder joint enable these activities in a smooth and coordinated manner. The shoulder joint complex is a combination of three joints viz; glenohumeral, acromioclavicular and sternoclavicular joints. In addition, scapulothoracic articulation forms a functional joint between the concave anterior surface of scapula and convex posterior thoracic wall. Together the complex creates smooth and well-coordinated movements of upper limbs ranging from the primitive activities of reaching and exploring the surroundings to the most intricate and dexterous movement patterns. Consequently shoulder joint is subjected to multiple injuries. These may result from trauma, immobilization, overuse injuries (occupational or sports related) or gradual degeneration. Shoulder joint disorders can be secondary to other systemic disorders inc-

Correspondence: Dr Sumeera Mattee, Classified Rehab Specialist, Combined Military Hospital, Mangla Pakistan

Received: 21 Oct 2019; revised received: 01 Jan 2020; accepted: 07 Jan 2020 luding various metabolic/endocrine disorders e.g. diabetes mellitus, neurologic disorders e.g. stroke, cardiac diseases, heart surgery, and malignancy ${ }^{1,2}$.

Shoulder joint disorders are the thirdcommonest kind of musculoskeletal disorders. The incidence of shoulder pain reporting in primary care per year is 14.7 per 1000 patients and the lifetime prevalence is $70 \%{ }^{3}$. Patients reporting with shoulder pain mainly fall into categories of adhesive capsulitis, rotator cuff syndrome, impingement syndrome, osteoarthritis, post-traumatic stiffness, and dislocations. However, there is no definite data on the prevalence of these different pathologies in general population.

There are diverse non-operative options available for treating shoulder joint disorders. These include intra-articular corticosteroids, intra-articular NSAIDs, viscosupplementation, platetet-rich plasma and capsular hydro-dilatation with normal saline ${ }^{4}$. Intra-articular platelet rich plasma has been found to have more sustained effects than corticosteroids however it is a costlier option and the pain relief is quite slow ${ }^{5}$. Corticosteroids have remained a mainstay of treatment in shoulder joint pathologies. Intra-articular corticosteroid injections reduce pain, inflammation and improve 
range of motion and hence contribute to early return of function ${ }^{6,7}$. Nevertheless, the risks and benefits of intraarticular corticosteroid injections are debatable. Intraarticular steroids have been linked to accelerated osteoarthritis in addition to short term adverse effects ${ }^{8}$. It is advisable to space subsequent injections by several weeks. Therefore not $>4$ injections are suggested in a year. Also if previous injections do not offer any relief, further injections are less likely to offer benefit. Corticosteroids are contraindicated in case of infections, malignancy or fractures. They are not to be given into ligaments/tendons due to risk of rupture. Post injection steroid flare has been observed within 24-36 hours secondary to crystal induced synovitis caused by precipitation of preservatives in steroid suspension. Other possible local side effects include, soft tissue/subcutaneous fat atrophy, and skin hypopig-mentation. Also in diabetic patients post injection surge in blood glucose levels occurs which may last for a week. Less common side effects include infection, vascular injury, and post injection neuritis ${ }^{6}$. Ketorolac (an NSAID) has been found to have a comparable efficacy with no observed damaging effect on the joint ${ }^{9}$. Thus it can be another tool in thearsenalwhen other options have proved ineffective for intra-articular injections for knee arthritis. To the best of our knowledge, there has been little research in Pakistan concerning the use of NSAIDs as intra-articular injection mixture. We hypothesized that intra-articular ketorolac injection would produce similar pain relief. Thus, the primary aim of this study was to compare the efficacy of ketorolac (an NSAID) with Depo-Medrol (methyl prednisolone i.e. a corticosteroids) inimproving range of motionfor various shoulder joint pathologies.

\section{METHODOLOGY}

This quais-experimental study (single-blind study) was carried out at the departments of internal and rehabilitation medicine, Combined Military Hospital Mangla, from November 2018 to May 2019. The Ethical review committee of the hospital reviewed the study proposal and found it to be ethically sound (EC ref no. 18/Trg/CMH Mgl dated 7 Nov 2018). After verbal informed consent, the individuals of age 18-75 years belonging to both genders who were diagnosed with a particular shoulder pathology and had shoulder pain for at least 3 months were selected through consecutive sampling covering ethical considerations as guided by the declarations of Helsinki. A sample size of 32 with 16 in each intervention group was calculated through an online sample size calculator while taking anticipated mean and standard deviation of group- 1 as 1.8 and 0.3 respectively, anticipated mean of group- 2 as 1.5 , with level of significance $(5 \%)$ and power of the test $(80 \%)^{10}$.

The selected shoulder pathologies were rotator cuff syndrome, adhesive capsulitis of the shoulder, and the shoulder impingement syndrome. Impingement syndrome was defined as shoulder pain with positive impingement sign (Neer's test or Hawkins-Kennedy test), painful arcduring active arm elevation, and positive empty can test ${ }^{11}$. A diagnosis of rotator cuff tendinosis was made if, on stressing the rotator cuff by applying a resistive force, the patient complained of pain in one or more portions of the rotator cuff with or without positive tests for impingement syndrome mentioned above ${ }^{12}$. Adhesive capsulitis was diagnosed if there was restriction of shoulder movements both passive and active in flexion, abduction, internal and external rotation with at least $50 \%$ in one movement compared with the normal side in the absence of bony restriction ${ }^{12,13}$. Patients with uncontrolled diabetes, fractures of bones comprising the shoulder joint, osteomyelitis, malignancy and deranged renal functions were excluded from the study.

Seventy-eight patients were identified as possible candidates as per the inclusion criteria and a total of 60 patients were finally selected for the study (figure).

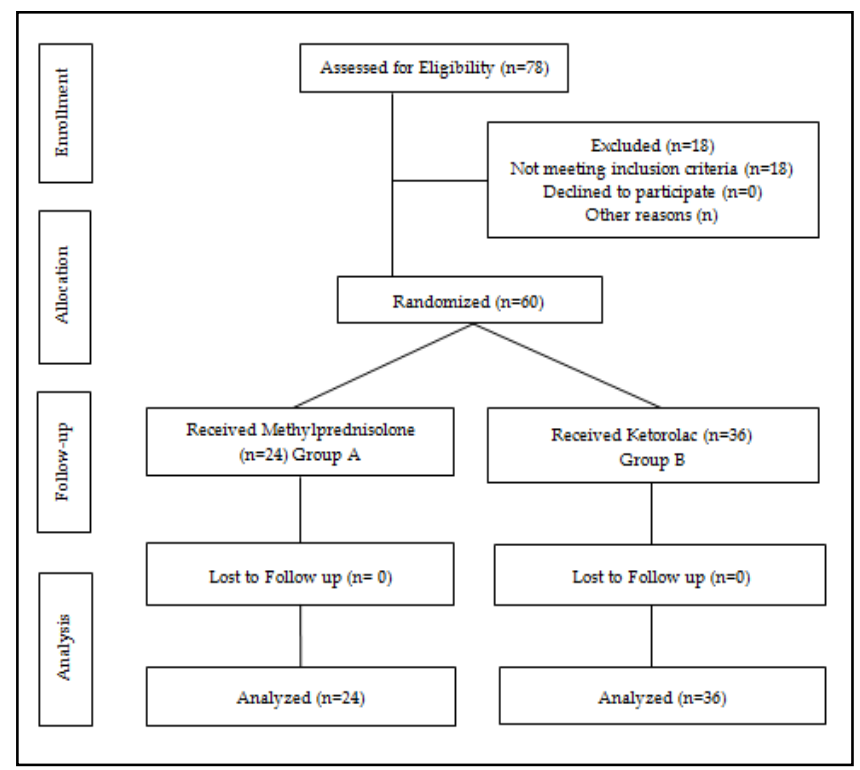

Figure: Flow chart.

Twenty-four $(40 \%)$ patients irrespective of shoulder pathology were randomly allocated thorough coin toss method to the Depo-Medrol group while $36(60 \%)$ were included in the ketorolac group. The ROM of the 
affected joint was measured through goniometry before injection and then at four weeks after the injection. A written proforma was filled for each patient that contained patient identification number, intervention group, age, primary shoulder pathology, and the measurements of ROM in flexion, extension, abduction, internal, and external rotation.

A $1 \mathrm{ml}(40 \mathrm{mg})$ of methyl prednisolone acetate with $2 \mathrm{ml}$ solution of $2 \% \mathrm{~W} / \mathrm{V}$ lignocaine (Lignocaine, Elite Pharma Limited, Lahore, Pakistan) via $5 \mathrm{ml}$ sterilized disposable syringe was injected into the affected shoulder of each patient with methyl prednisolone acetate injection while $1 \mathrm{ml}(30 \mathrm{mg})$ of Ketorolac (Toradol, Roche Pakistan limited, Karachi, Pakistan) with $2 \mathrm{ml}$ solution of $2 \% \mathrm{~W} / \mathrm{V}$ lignocaine (Lignocaine, Elite Pharma Limited, Lahore, Pakistan) via $5 \mathrm{ml}$ sterilized disposable syringe was injected for each patient with ketorolac injection. The blind technique for shoulder injection i.e. without ultrasound or fluoroscopic guidance was used in both groups. For all patients, posterior approach glenohumeral injection was administered. A single operator performed the injection procedures in both groups. The operator was a consultant physiatrist with seven years of experience in performing pain procedures.

All inductees were shifted on a combination of paracetamol $(650 \mathrm{mg}$ and orphenadrine citrate $(50 \mathrm{mg})$ (Nuberol Forte, Searle Pakistan Limited, Karachi, Pakistan) twice daily orally and local application of piroxicam gel 0.5\% w/w (Pcam, Merck Private Limited, Karachi, Pakistan) four times a day. After the injection, a comprehensive rehabilitation plan comprising of therapeutic exercises particular to the disease was given to all patients in both groups. All exercises in the we provided the patients a feasible belated period of one week, however, the measurement results were reflected in the same category of 4 week post-treatment. To encourage timely follow-up, we offered the recruits appointment-free checkup for the next 6 months if they reported at the appropriate time. Luckily, it worked and all recruits reported for the follow up in time and there was no dropout.

The Statistical Package for Social Sciences version 20 (IBM Corp., Armonk, NY, USA) was used for all analyses. All values were measured for the groups before start of intervention, and after 4 weeks of intervention. For variables of age and ROM, means and standard deviations were calculated. The improvements in ROM between the two groups were analyzed using independent sample or paired sample t-tests where appropriate. A $p$-value $\leq 0.05$ was considered statistically significant.

\section{RESULTS}

Of 60 patients finally selected, 40 (66.7\%) were male and $20(33.3 \%)$ were female. The mean age of the sample was $49 \pm 11$ years (range: $27-70$ years). Thirty $(50 \%)$ patients had adhesive capsulitis, $24(40 \%)$ had rotator cuff syndrome and $4(10 \%)$ had impingement syndrome. The demographics for the two groups are compared in table-I. Twenty-four patients received methyl prednisolone acetate injection while 36 received ketorolac injection. The ROM in flexion, extension, abduction, internal rotation, and external rotation before and after treatment are given in table-II. Each group had a significant gain in ROM in flexion, extension, abduction, internal, and external rotation after treatment (table-II).

Table-I: Comparison of demographics among the two treatment groups frequency.

\begin{tabular}{l|c|c}
\hline Variables & $\begin{array}{c}\text { Group A (Methyl Prednisolone } \\
\text { Acetate Injection) (n=24) }\end{array}$ & $\begin{array}{c}\text { Group B (Ketorolac Injection) } \\
\text { (n=36) }\end{array}$ \\
\hline Mean age & $55 \pm 9$ years (range: 38-70 years) & $45 \pm 10$ years (range: 27-62 years) \\
\hline Gender & $14(58.3 \%)$ & $26(72.2 \%)$ \\
\hline Male & $10(41.7 \%)$ & $10(27.8 \%)$ \\
Female & $12(50 \%)$ & $18(50 \%)$ \\
\hline Shoulder Pathology & $10(41.7 \%)$ & $14(38.9 \%)$ \\
Adhesive Capsulitis & $2(8.3 \%)$ & $4(11.1 \%)$ \\
Rotator Cuff Syndrome & \multicolumn{2}{|c}{} \\
Shoulder Impingement Syndrome & \multicolumn{2}{|c}{} \\
\hline
\end{tabular}

plan were home based as most of our recruits were far dwellers and found it difficult to come to the rehabilitation center on daily basis. The same investigator took the ROM measurements before and after four weeks of intervention through goniometer. For the follow up,
There was no significant difference in the mean gain in flexion, extension, abduction, internal or external rotation between both groups $(p=0.224, p=0.261$, $p=0.884, p=0.238$, and $p=0.584$ respectively) (table-III). 
Table-II: Pre and post intervention mean values and their comparison for range of motion in flexion, extension, abduction, internal rotation, and external rotation for each group.

\begin{tabular}{|c|c|c|c|c|c|c|}
\hline \multirow{2}{*}{ Variables } & \multicolumn{2}{|c|}{$\begin{array}{l}\text { Group A (Methyl Prednisolone } \\
\text { Acetate Injection) }(n=24)\end{array}$} & \multirow{2}{*}{$p$-value } & \multicolumn{2}{|c|}{$\begin{array}{c}\text { Group B (Ketorolac Injection) } \\
(\mathrm{n}=36)\end{array}$} & \multirow{2}{*}{$p$-value } \\
\hline & Pre- Treatment & $\begin{array}{l}\text { Four Weeks Post } \\
\text { Treatment }\end{array}$ & & Pre-Treatment & $\begin{array}{c}\text { Four Weeks } \\
\text { Post Treatment }\end{array}$ & \\
\hline & Mean $\pm S D^{*}$ & Mean \pm SD & & Mean \pm SD & Mean \pm SD & \\
\hline Flexion & $95.4 \pm 31.4$ & $117.1 \pm 29.2$ & $<0.001$ & $106.7 \pm 28.1$ & $135.6 \pm 29.4$ & 0.001 \\
\hline Extension & $36.2 \pm 15.9$ & $45.4 \pm 11.7$ & $<0.001$ & $43.3 \pm 12.3$ & $55.1 \pm 6.7$ & $<0.001$ \\
\hline Abduction & $87.5 \pm 35.8$ & $115.4 \pm 32.9$ & $<0.001$ & $102.6 \pm 30.9$ & $131.1 \pm 27.6$ & $<0.001$ \\
\hline Internal Rotation & $24.6 \pm 15$ & $41.2 \pm 16.4$ & 0.007 & $34.2 \pm 15.5$ & $55.8 \pm 19.6$ & $<0.001$ \\
\hline External Rotation & $38.3 \pm 28.9$ & $54.6 \pm 27.9$ & $<0.001$ & $50 \pm 23.8$ & $68.1 \pm 22$ & $<0.001$ \\
\hline
\end{tabular}

N: Frequency *SD: Standard deviation.

Table-III: The mean improvement in range of motion in flexion, extension, abduction, internal and external rotation.

\begin{tabular}{l|c|c|c}
\hline Variables & $\begin{array}{c}\text { Group A (Methyl Prednisolone } \\
\text { Acetate) }(\mathbf{n = 2 4 )} \text { Mean (SD*) }\end{array}$ & $\begin{array}{c}\text { Group B (Ketorolac) } \\
\text { (n=36) Mean (SD) }\end{array}$ & $\boldsymbol{p}$-value \\
\hline Mean increase in flexion & $21.7(17.6)$ & $28.9(27.8)$ & 0.224 \\
\hline Mean increase in extension & $9.2(7.8)$ & $11.8(10)$ & 0.261 \\
\hline Mean increase in abduction & $27.9(13.3)$ & $28.5(17.4)$ & 0.884 \\
\hline Mean increase in internal rotation & $16.7(15.2)$ & $21.7(16.8)$ & 0.238 \\
\hline Mean increase in external rotation & $16.2(12.7)$ & $18.1(12)$ & 0.584 \\
\hline
\end{tabular}

$N$ : Frequency ${ }^{*}$ SD: Standard deviation.

\section{DISCUSSION}

The results of our study are comparable to earlier studies which reveal a similar efficacy of intra-articular steroids and NSAIDs. Min et al ${ }^{14}$, compared ketorolac to methylprednisolone in 32 patients with subacromial impingement syndrome when used as a single dose subacromial injection. Their study reported comparable efficacy of both these agents in terms of improvement in the University of California at Los Angeles (UCLA) Shoulder Scores during 4 week follow-up. Tahiri et al ${ }^{15}$, reported that a single injection of $60 \mathrm{mg}$ ketorolac resulted in equivalent improvements in outcome compared to a single injection of $40 \mathrm{mg}$ methylprednisolone for the treatment of subacromial impingement when assessed at 12 weeks. In a retrospective comparative study by Park et alli, it was reported that the treatment of osteoarthritis of the hip with intraarticular ketorolac injection was as effective as that with intra-articular corticosteroid injection. $\mathrm{Xu}$ et al ${ }^{9}$, carried out a pilot-controlled clinical study to assess the effect of ketorolac in intra-articular injection analgesia for postoperative pain in patients undergoing shoulder arthroscopy. They concluded that the combination of ketorolac and a multimodal drug regime (ropivacaine, morphine, and triamcinolone acetonide) resulted in improved post-surgical pain scores. They also proposed further prospective controlled trials to allow for definite treatment recommendations. In another study conducted by Bellamy et al ${ }^{17}$, the economic impact of ketorolac was compared with corticosteroid intra-articular knee injection for osteoarthritis. Accor- ding to them, pain relief was similar between the two injectates. Ketorolac knee injection was found safe and effective with a cost savings percentage difference of $143 \%$ when compared with corticosteroid.

The side effects of intra-articular steroids have been confirmed in various studies. In a study by Kew et $a^{18}$, in a cohort of patients who underwent arthroscopic surgery for rotator cuff repair or subacromial decompression, the risk of infection after 1 month was higher in patients who had received intra articular corticosteroid injection compared to the control group who did not receive any such injection. Similarly Baverel et al ${ }^{19}$, found that the re-tear rate of rotator cuff in patients who received corticosteroid injection after arthroscopic repair of the rotator cuff was greater than the patients who did not receive post-operative corticosteroid injection.

Assuming that both NSAIDs and corticosteroids function by locally decreasing inflammation, this study provides evidence of equivalent if not superior results of injectable ketorolac. Improvement in function and satisfaction are directly associated with the patient's ability to strengthen the rotator cuff. Practitioners may utilize this as a possible short-term alternative for nonoperative treatment of subacromial impingement syndrome. Therefore with a similar efficacy and less side effects of intra-articular NSAIDs as compared to corticosteroids proved by this body of evidence, clinicians and patients can make a sound decision regarding the choice of treatment modality. 
We used the coin method for randomization. Currently, in randomized study designs, the coin method is generally discouraged because it results in unequal distribution of subjects in both groups. Secondly, the results are based upon the immediate improvements in shoulder ROM at 4 weeks post injection. Long term assessment could not be done due to failure to follow up. Thirdly, we used blind technique for injection. Injections under ultrasound guidance might have produced even better results.

\section{CONCLUSION}

Both methylprednisolone and ketorolac have equivalent outcomes when used as intra-articular injectates in shoulder disorders on the basis of improvement in the range of motion of shoulder. Therefore, ketorolac can be considered as alternative drug where corticosteroids are contraindicated, provided that the patient has no past history of allergy to NSAIDs and has optimal renal functions.

\section{CONFLICT OF INTEREST}

This study has no conflict of interest to be declared by any author.

\section{REFERENCES}

1. Akhlaq U, Ayaz SB, Akhtar N, Khan AA. Frequency and intensity of shoulder pain after stroke: A hospital based study. Pak Arm For Med J 2016; 66(1): 71-74.

2. Hussain A, Ayaz SB, Mumtaz SN, Ahmad K. Characteristics and predictors of chronic non-anginal postoperative pain after openheart surgery in a cohort of pakistani population. Pak Heart J 2015; 48(2): 1-8.

3. Singh S, Gill S, Mohammad F, Kumar S, Kumar D, Kumar S. Prevalence of shoulder disorders in tertiary care centre. Int J Res Med Sci 2015; 3(4): 917-20.

4. Tran K, Loshak H. Intra-articular hyaluronic acid for visco supplementation in osteoarthritis of the hand, shoulder, and temporomandibular joint: A rev clin effect safety 2019 [Internet] Available at: https://europepmc.org/article/nbk/nbk546787? report= printable\&client=bot\&client=bot\&client=bot (Accessed at: 15Jan-2019)

5. Saif DS, Serag DM, El Tabl MA. Comparative study between platelet-rich plasma injection and steroid injection in mildmoderate shoulder osteoarthritis and their relation to quality of life. Egypt Rheumatol Rehabilit 2019; 46(1): 55-58.

6. Nichols AW. Complications associated with the use of corticosteroids in the treatment of athletic injuries. Clin J Sport Med 2005; 15(5): E370-75.
7. Sharma SP, Bærheim A, Moe-Nilssen R, Kvåle A. Adhesive capsulitis of the shoulder, treatment with corticosteroid, corticosteroid with distension or treatment-as-usual; a randomised controlled trial in primary care. BMC musculoskeletal disorders 2016; 17(1): 232-35.

8. Kompel AJ, Roemer FW, Murakami AM, Diaz LE, Crema MD, Guermazi A. Intra-articular corticosteroid injections in the hip and knee: Perhaps not as safe as we thought?. Radiol 2019; 293(3): 656-63.

9. Xu J, Qu Y, Li H, Jiang T, Zheng C, Wang B, et al. effect of ketorolac in intra-articular injection analgesia for postoperative pain in patients undergoing shoulder arthroscopy: a pilot-controlled clinical study. J Pain Res 2019; 12(1): 417-20.

10. Lee HJ, Lim KB, Kim DY, Lee KT. Randomized controlled trial for efficacy of intra-articular injection for adhesive capsulitis: ultrasonography-guided versus blind technique. Arch Phys Med Rehabil 2009; 90(1): 1997-2002.

11. Michener LA, Yesilyaprak SSS, Seitz AL, Timmons MK, Walsworth MK. Supraspinatus tendon and subacromial space parameters measured on ultrasonographic imaging in subacromial impingement syndrome. Knee Surgery, Sports Traumatol Arthroscopy 2015; 23(2): 363-69.

12. Ostor A, Richards C, Prevost A, Speed C, Hazleman B. Diagnosis and relation to general health of shoulder disorders presenting to primary care. Rheumatol 2005; 44(6): 800-805.

13. Çalış HT, Karabaş Ç, Güler E. Effects of Platelet-rich Plasma Injection on Adhesive Capsulitis: An Interventional Case Series. Erciyes Med J/Erciyes Tip Dergisi 2019; 41(1): p102-106.

14. Min KS, Pierre PS, Ryan PM, Marchant BG, Wilson CJ, Arrington ED. A double-blind randomized controlled trial comparing the effects of subacromial injection with corticosteroid versus NSAID in patients with shoulder impingement syndrome. J Shoulder Elbow Surg 2013; 22(5): 595-601.

15. Taheri P, Dehghan F, Mousavi S, Solouki R. Comparison of Subacromial Ketorolac Injection versus Corticosteroid Injection in the Treatment of Shoulder Impingement Syndrome. J Res Phar Pract 2017; 6(4): 223-25.

16. Park KD, Kim TK, Bae BW, Ahn J, Lee WY, Park Y. Ultrasound guided intra-articular ketorolac versus corticosteroid injection in osteoarthritis of the hip: a retrospective comparative study. Skeletal Radiol 2015; 44(9): 1333-40.

17. Bellamy JL, Goff BJ, Sayeed SA. Economic impact of ketorolac vs corticosteroid intra-articular knee injections for osteoarthritis: a randomized, double-blind, prospective study. J Arthroplasty 2016; 31(9): 293-97.

18. Kew ME, Cancienne JM, Christensen JE, Werner BC. The timing of corticosteroid injections after arthroscopic shoulder procedures affects postoperative infection risk. Am J Sports Med 2019; 47(4): 915-21.

19. Baverel L, Boutsiadis A, Reynolds RJ, Saffarini M, Barthélémy R, Barth J. Do corticosteroid injections compromise rotator cuff tendon healing after arthroscopic repair. JSES Open Access 2018; 2(1): 54-59. 